Military Technical College, Kobry El-Kobbah, Cairo, Egypt

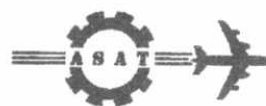

$9^{\text {th }}$ International Conference

On Aerospace Sciences \&

Aviation Technology

\title{
TWO-DIMENSIONAL ANALYSIS OF A STRAIGHT FIN IN A FLOW FIELD WITH A LOCATION DEPENDENT HEAT TRANSFER COEFFICIENT
}

\section{ABSTRACT}

\section{S. A. ABDEL-MONEIM}

Two-dimensional modeling and numerical analysis of a straight fin in a convectiveradiative environment are presented. The energy equation is adapted to model a fin with rectangular profile in a flow field with streamwise variable heat transfer coefficient. The finite difference technique is used to discretize this equation and a numerical solution is obtained via an iterative scheme. Temperature profiles of the fin surface, fin efficiency and the heat dissipated per unit mass of the fin material are predicted at different flow velocities and thermal conditions. Also, a method is proposed to reduce the weight of the straight fin and in accordance to minimize the flow- pumping power by providing a nonuniform fin-height profile. This optimum profile is estimated by tracing the local effective fin heights that maximize the heat dissipated per unit mass. New correlations based on the predicted results are obtained for the fin effective height, fin efficiency and heat dissipated per unit mass as functions of the flow velocity, fin geometry, fin material-properties and the operating base temperature.

\section{KEYWORDS: Two-dimensional fin, Fin optimization, Fin performance}

\section{NOMENCLATURE}

Unless otherwise stated SI system of units was used for the dimensional parameters:

$A_{p} \quad$ corrected fin profile area, $A_{p}=L_{c}{ }^{*} b \quad M \quad$ number of nodes in $x$ direction

$b$ fin thickness $N$ number of nodes in y direction

C coefficient for Eqs.(13-16) q heat transfer rate

$\mathrm{H}$ fin height

h heat transfer coefficient

i,j nodal identification numbers

k thermal conductivity

$\mathrm{L} \quad$ fin streamwise length

$L_{c}$ corrected fin-height, $L_{c}=H+b / 2$

$T$ temperature

U freestream flow velocity

$\mathrm{V}$ fin material volume

$x \quad$ streamwise coordinate

y normal coordinate

* Associate Prof., Mech. Eng. Dept., Faculty of Eng. (Shoubra), Zagazig University 108 Shoubra St., Cairo, Egypt, E-mail: sayed_moneim@hotmail.com 


\section{Greek Letters:}

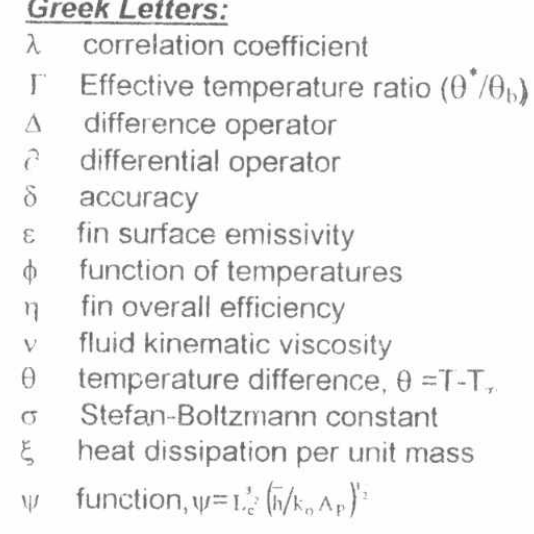

\section{Dimensionless Terms:}

Nu Nusselt number

Pr Prandtl number

Re Reynolds number

VR volume reduction ratio

\section{Superscripts:}

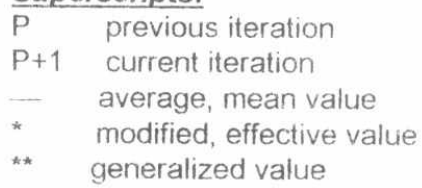

\section{Subscripts:}

$\begin{array}{ll}\text { I, II } & \text { fin efficiency limits } \\ \text { b } & \text { fin base } \\ \text { Cond. } & \text { conduction heat transfer } \\ \text { Conv. } & \text { convection heat transfer } \\ i, j & \text { node position } \\ \text { L } & \text { based on the fin length } \\ \text { o } & \text { property value at } 300 \mathrm{~K} \\ \mathrm{R} & \text { reference fin parameters } \\ \text { Rad. } & \text { radiation heat transfer } \\ S & \text { fin solid material } \\ x & \text { local } x \text {-value } \\ y & \text { local } y \text {-value } \\ \infty & \text { ambient }\end{array}$

\section{INTRODUCTION}

Fins are widely used in thermal systems to augment the heat transfer especially for systems operating at elevated thermal loading and cooled with gaseous fluids. There are many enhanced variants of fin patterns such as Louver, wavy and slit fins patterns, yet straight fins are still by far the most popular fin pattern. This is because of its superior reliability, simplicity in manufacturing and its relatively lower friction coefficient [1]. The pioneering published contributions of Murray [2] and Gardner [3] are considered the premier heat transfer analysis for the different fin patterns. Many efforts have been devoted to optimize the straight fin design. Kraus [4] summarized most of the relevant published studies that investigated the use of plain fins in engineering and industrial applications.

Experiments were carried out by Leong and Kooi [5] to study the temperature dependence of the heat transfer coefficient (HTC) in a steady-state natural convection from vertical rectangular mild steel and aluminum fins using laser holographic interferometry. It was found that, depending on the fin material and base temperature, the local HTCs vary along the fin and reach maximum values at distances measured from the base ranging from 0.22 up to 0.48 of the fin height. The effect of the temperature-dependent HTC for a natural flow field on the performance of fins was reported in $[6,7]$. Fins of rectangular, triangular, and parabolic profiles in addition to cylindrical, conical and parabolic spines were theoretically-numerically analyzed by Laor and Kalman [6]. The performance and the optimum dimensions of these fins were correlated with the different investigated parameters. Also, the optimum dimensions of rectangular and cylindrical-pin fins in an environment of temperature dependent HTC were analyzed by Yeh [7]. The fin volume was fixed to obtain the aspect ratio of the uniform area cross-section fins that maximizes the heat 
transfer rate. The analysis showed that an optimum aspect ratio was not found for a fin with heat transfer from the tip at a large fin volume or a large heat transfer coefficient at the fin base. However, an optimum aspect ratio for an insulated-tip fin was found. The optimum aspect ratio of a fin was found to be higher for a fin with an insulated tip and decreases on increasing the rate of heat transfer from the tip.

Optimization of shape and spacing of straight fins, cooled by a fluid in laminar flow. was attempted by Fabbri, [8] and Garg and Velusamy [9]. Fabbri, [8] used a finite element model to determine the velocity and temperature distributions, which were employed in a genetic algorithm to find the fin thickness profile, which make the heat transfer rate as high as possible under different conditions. Garg and Velusamy [9] analyzed a straight fin at different Prandtl numbers ranging from 0.1 up to 100 using an empirical correlation for the local HTC of the form $\left(h_{x} \propto x^{-0.5}\right)$.

The influence of a fin thermal, physical and geometrical parameters on the optimum dimensions of fins in a flow with a relatively high velocity, were reported in [10-13]. A closed-form model for the second-law-based thermo-economic optimization of constant cross-sectional area fins was conducted by Shuja and Zubair [10]. An approach was applied in which different monetary values were attached to the irreversible losses caused by the fin-flow temperature difference as well as the pressure drop in the flow passage The optimal length of a rectangular profile longitudinal fin with convective-radiative tip and variable thermal conductivity, was investigated by $\mathrm{Yu}$ and Chen [11]. The governing nonlinear conduction equation was solved using the Taylor transformation method. It was found that the temperature of the fluid on the other side of the primary surface is an important optimization parameter. Prasad and Gupta [12] proposed a method for reducing the weight of a straight rectangular fin by providing a semicircular cut at its tip. Finite element analysis was used to compute the temperature distributions and estimate the fin efficiency values. A typical value of fin-efficiency for the proposed fin was found to be $66.85 \%$ at a Biot number of 0.1048 and a cut-size ratio of 0.798 . Also, an efficiency of $68.05 \%$ was found for an array of these fins, when the pitch was equal to twice the thickness. Morega and Bejan [13] tried to solve the problem of hot spots of an electronic module by allowing the fin thickness and height to increase in the flow direction. The hot spots decreased by about $15 \%$ when the thickness of the fin of constant height increased as $x^{0.42}$. However, a reduction in the hot spots of $30 \%$ was found when the height of a constant thickness fin increased linearly in the flow direction. Kang. [14] performed a comparison between the heat loss of the asymmetric triangular fin and the asymmetric trapezoidal fins, which have various slopes of the fin's upper lateral side. The relation between the slope factor of the fin and the non-dimensional fin length for equal amount of heat loss was obtained In this study the base temperature, thermal conductivity of fin's material and the heat transfer coefficient were assumed constant.

In fact, much use of the previous models that analyze fins have been performed based on the Murray and Gardner [2] assumptions as follows:

1- Uniform and constant heat transfer coefficient all over the fin surface.

2- The fin material is isotropic with temperature independent properties.

3-Two-dimensional fin geometry, i.e. lins with small thickness such that the

temperature gradient in the direction normal to the fin surface is negligible.

4- Heat flow without internal heat generation. 
5- Steady state heat transfer mechanism.

6- The temperature of the fin base is constant and uniform.

7- The temperature of the surroundings is constant.

In the real world applications, two factors that were not considered in the previous analysis may affect the fin behaviour. First, the heat transfer coefficient is a location dependent as well as a temperature dependent parameter. Second, most of the real materials from which the fins are made have temperature dependent properties Therefore, the present model was developed to take into considerations the effect of these two factors on the fin behavior and the main objectives of the present study are:

1) to predict precisely the behaviour of straight fins by two-dimensional modeling.

2) to modify the predictions by considering the variation in the local heat transfer coefficient.

3) to provide engineers with correlations for fin performance much close to reality

4) to optimize fin material and fluid flow pumping power consumption by providing optimum fin-geometries.

\section{Theoretical Modeling}

A two-dimensional model of a straight fin in a convective-radiative environment with location dependent HTC is presented. The geometry of the proposed fin model is shown in Fig.(1). The flow was considered two-dimensional in a plane orthogonal to the fin surface. This leads to a flow regime of a location dependent HTC. Also, the temperature variation within the fin thickness was assumed negligible in comparison with that experienced in the other two-dimensions. This was established by limiting the fin thickness such that Biot number based on the fin thickness, b, was limited to $\mathrm{Bi}=\frac{\mathrm{b} \bar{h}}{\mathrm{k}_{\mathrm{s}}} \leq 0.1$, Bejan [15]. Also, a linear temperature dependent thermal conductivity was considered as: $k_{s}=k_{0}+\alpha T$, where $k_{0}$ is the thermal conductivity of the fin material evaluated at $300 \mathrm{~K}$ and $\alpha$ is a constant that characterizes the fin material and it is evaluated by fitting the data values tabulated in Incropera and DeWitt [16].

\subsection{Governing Equation}

The governing equation is derived for the two-dimensional fin geometry shown in Fig.(1) by performing an energy balance (heat balance) for a fin element as follows:

$$
q_{x}+q_{y}=q_{x+d x}+q_{y+d y}+q_{\text {conv }}+q_{\text {Rad }}
$$

where,

$q_{x}, q_{y}$ are the inlet conduction to the two-dimensional fin element in $x$ and $y$ directions, respectively.

$q_{x+d x}, q_{y+d y}$ are the outlet conduction from the fin element in $x$ and $y$ directions, respectively.

$q_{\text {conv. }} \quad$ is the heat transferred by convection through the two sides of the fin element. 

element.

The conduction heat transfer $q_{x}$ and $q_{y}$ are simply calculated from Fourier's law as:

$$
\text { and } \begin{aligned}
q_{x} & =-k_{s} \Delta y b \frac{\partial T}{\partial x} \\
q_{y} & =-k_{s} \Delta x b \frac{\partial T}{\partial y}
\end{aligned}
$$

Also, $q_{x+d x}$ and $q_{y+d y}$ are approximated by the first two terms of Taylor's expansion as

$$
q_{x-d x}=q_{x}+\frac{\partial}{\partial x}\left(-k_{s} \frac{\partial T}{\partial x}\right) \Delta x \Delta y b
$$

and

$$
q_{y+d y}=q_{y}+\frac{\partial}{\partial y}\left(-k_{s} \frac{\partial T}{\partial y}\right) \Delta x \Delta y b
$$

where the fin temperature is modeled as a two dimension function, $T=T(x, y)$

The convection heat transfer qconv. is calculated from Newton's law of cooling as

$$
q_{\text {corv. }}=2 h_{x} \Delta x \Delta y\left(T-T_{r}\right)
$$

Also, the radiation heat transfer $q_{\text {Rad }}$ is estimated from Stefan-Boltzman's law with the two sides of the fin were considered gray surfaces with a constant emissivity $\varepsilon$ as:

$$
\mathrm{q}_{\mathrm{Rad} .}=2 \varepsilon \sigma \Delta \mathrm{x} \Delta \mathrm{y}\left(\mathrm{T}^{+}-\mathrm{T}_{* .}^{+}\right)
$$

where the local heat transfer coefficient $h_{x}$ is calculated as a function of the local Reynolds number $\mathrm{Re}_{\mathrm{x}}$ from existing correlations, [16] as and

$$
N u_{x}=0.332 \operatorname{Re}_{x}^{0.5} \mathrm{Pr}^{0.333} \text { for laminar flow regime, } \mathrm{Re}_{\mathrm{x}} \leq 5 \times 10^{5}
$$

$$
\mathrm{Nu}_{x}=0.0296 \mathrm{Re}_{x}^{0.8} \mathrm{Pr}^{0.333} \text { for turbulent flow regime, } \mathrm{Re}_{\mathrm{x}}>5 \times 10^{5}
$$

where, $\operatorname{Re}_{\mathrm{x}}$ is calculated based on the streamwise location $\mathrm{x}$ as: $\operatorname{Re}_{\mathrm{x}}=\frac{U \mathrm{x}}{\mathrm{v}}$

Substituting Eqs. (2,3,4 and 6) in Eq. (1) and rewriting yields;

$$
\frac{\partial}{\partial x}\left(k_{s} \frac{\partial T}{\partial x}\right)+\frac{\partial}{\partial y}\left(k_{s} \frac{\partial T}{\partial y}\right)-\frac{2 h_{x}}{b}\left(T-T_{\infty}\right)-\frac{2 \varepsilon \sigma}{b}\left(T^{4}-T_{y}^{4}\right)=0
$$

To simplify the problem and to avoid the non-linearity associated with the first two terms of Eq.(7), $\mathrm{k}_{\mathrm{s}}$ is assumed spatially constant although it is numerically treated as 
a linear function of temperature. Thus yields the following second order nonlinear partial differential equation

$$
\frac{\partial^{2} T}{\partial x^{2}}+\frac{\partial^{2} T}{\partial y^{2}}-\frac{2 h_{x}}{b k_{s}}\left(T-T_{,}\right)-\frac{2 \varepsilon \sigma}{b k_{s}}\left(T^{4}-T^{4}\right)=0
$$

\subsection{Computational Domain and Boundary Conditions}

Equation (8) is valid within the two-dimensional domain $(0 \leq x \leq L, 0 \leq y \leq H)$ and subjected to the following boundary conditions at the two sides of the fin and at the fin base and tip.

i) The flow inlet and exit sides at $(x=0,0 \leq y \leq H)$ and at $(x=L, O \leq y \leq H)$,

respectively, are considered convective-radiative boundaries

$\left(q_{\text {cond }}=q_{\text {conv }}+q_{\text {rad }}\right)$. Therefore

$$
-k_{s}\left(\frac{\partial T}{\partial x}\right)_{x, y}=h_{x}\left(T-T_{\ldots}\right)+\varepsilon \sigma\left(T^{4}-T^{4}\right)
$$

ii) At the fin tip a convective-radiative boundary condition is also assumed and the following equation is applied at $(y=H, O \leq x \leq L)$

$$
-k_{s}\left(\frac{\partial T}{\partial y}\right)_{x, y}=h_{x}\left(T-T_{,},\right)+\varepsilon \sigma\left(T^{4}-T^{4}\right)
$$

iii) At the fin base $(y=0,0 \leq x \leq L)$ a uniform temperature of $T_{b}$ is assumed and

$$
T(x, 0)=T_{b} \quad \text { for } 0 \leq x \leq L
$$

\subsection{Discretization Scheme and Numerical Procedure}

The finite difference technique with a central difference model is used to discretize both the governing equation and the boundary conditions within the computational domain. The governing equation, Eq.(8) becomes;

$$
\frac{T_{i-1, j}+T_{i+1, j}-2 T_{i, j}}{\Delta x^{2}}+\frac{T_{i, j}+T_{i, j+1}-2 T_{i, j}}{\Delta y^{2}}-\frac{2 h_{i}}{b k_{s}}\left(T_{i, j}-T_{,}\right)-\frac{2 \varepsilon \sigma}{b k_{s}}\left(T_{i, j}^{4}-T_{, j}^{4}\right)=0
$$

Equation (12) is valid for the interior nodes only (where, $i=2,3, \ldots \mathrm{N}-1$ and $j=2,3, \mathrm{M}-1$ )

To treat the non-linear term $\left(\mathrm{r}_{1,}^{+},-\mathrm{T}_{\alpha}^{1}\right)$ a simple mathematical trick is performed this by expanding it into a linear part and a non-linear part as: $\left(T_{1, j}^{4}-T_{1}^{+}\right)=\left(T_{1},-T_{,}\right) \phi_{i, j}$ where, $\phi_{i, j}$ is the non-linear part and it is a function of $\left(T_{i, j}, T_{,}\right)$and reads, $\phi_{t, j}=\left(\mathrm{T}_{i, 1}+\mathrm{T}_{\sigma}\right)\left(\mathrm{T}_{i, j}^{2}+\mathrm{T}_{w}^{2}\right)$. This make it possible to transform the non-linear equation Eq.(12) into a semi-explicit form as: 
where,

$$
\theta_{i, j}^{p+1}=\frac{1}{C_{i, j}}\left[\frac{1}{1 x^{2}}\left(\theta_{i \cdot 1, j}^{p}+\theta_{i-1, j}^{p}\right)+\frac{1}{i y^{2}}\left(\theta_{i, j+1}^{p}+\theta_{i, j}^{p}\right)\right]
$$

$$
\begin{aligned}
& \theta_{i, j}=\left(T_{i, j}-T_{\alpha}\right) \\
& C_{i, j}=\left(\frac{2}{\Delta x^{2}}+\frac{2}{\Delta y^{2}}+\frac{2 h_{i}}{k_{s} b}+\frac{2 \varepsilon \sigma}{k_{s} b} \phi_{i, j}^{p}\right)
\end{aligned}
$$

and the indices $\mathrm{P}+1$ and $\mathrm{P}$ are related to the current and the previous iterations, respectively.

The boundary conditions Eqs.(9, 10, and 11) are also, discretized, transformed into semi-explicit forms and applied at their relative boundaries as follows

a) At the flow inlet side (where, $i=1$ and $j=1,2, \ldots . M$ );

$$
\theta_{1, j}^{p: 1}=\frac{\theta_{2, j}^{p}}{C_{1, j}}
$$

where,

$$
C_{1, j}=\left(1+\frac{h_{1} \Delta x}{k_{s}}+\frac{\varepsilon \sigma \Delta x}{k_{s}} \phi_{1, j}^{p}\right)
$$

b) At flow exit side (where, $i=N$ and $j=1,2, \ldots . M$ ),

$$
\theta_{N, j}^{P+1}=\frac{\theta_{N-1 . j}^{P}}{C_{N, j}}
$$

where,

$$
C_{N, j}=\left(1+\frac{h_{N} \Delta x}{k_{s}}+\frac{\varepsilon \sigma \Delta x}{k_{s}} \phi_{N, j}^{p}\right)
$$

c) At the fin tip (where, $i=1,2, \ldots \mathrm{N}$ and $\mathrm{j}=\mathrm{M}$ )

$$
\theta_{i, M}^{P_{+1}}=\frac{\theta_{i, M-1}^{p}}{C_{i, M}}
$$

where, $\quad C_{i, M}=\left(1+\frac{h_{1} \Delta y}{k_{s}}+\frac{\varepsilon \sigma \Delta y}{k_{s}} \phi_{i, M}^{p}\right)$

d) At the fin base (where, $i=1,2, \ldots \mathrm{N}$ and $j=1$ )

$$
\theta_{\mathrm{i}, 1}=\theta_{\mathrm{b}}, \quad \mathrm{i}=1,2, \mathrm{~N}
$$


The system of semi-explicit equations, Eqs.(13 to 17), is numerically solved resuiting in the temperature distribution within the computational domain $\langle i=1,2, \ldots \mathrm{N}$ and $\mathrm{j}=1,2, \ldots \mathrm{M})$. This is carried out via an iterative technique according to the following stepwise procedure

1) A starting temperature distribution is guessed as: $\theta_{i, i}^{P}=\theta_{b} / 2$

2) The average fin surface temperature is calculated by, $\theta_{A v}^{p}=\frac{1}{N M_{1}} \sum_{i, 1, N}^{i, M} \theta_{i, j}^{p}$ and the fin material properties are evaluated at this average temperature.

3) The local heat transfer coefficient is estimated according to the flow regime using the correlations Eqs. (6-a and 6-b) and the flow thermal properties are taken at the mean film temperature, $T_{i}^{P}=\left(\frac{\theta_{A v}^{P}+2 T_{w}}{2}\right)$.

4) The previous temperatures are updated by the new values $\left(00_{1.1}^{+, 1}\right)$ which are obtained from the solution of Eqs.(13 to 17).

5) The updated average temperature $\left(\theta_{A i}^{p+1}\right)$ is calculated as in step 2.

6) The convergence is checked, by comparing the average values of step 2 and step 5 to a certain accuracy as $\frac{\left|\theta_{\mathrm{Av}}^{P .1}-\theta_{\mathrm{Av}}^{\rho}\right|}{\theta_{\mathrm{Av}}^{P}} \leq \delta$.

7) If the convergence is not established, then the old values $\theta_{i, j}^{P}$ are replaced with the new values $\theta_{i, j}^{p+1}$ and then steps 2-7 are repeated.

8) If the accuracy is verified then the solution is converged and hence the fin efficiency $(\eta)$ and the heat dissipated from the fin per unit mass $(\xi)$ are calculated as: $\eta=\frac{q_{1}}{q_{\max } \text {. }}$ and $\xi=\frac{q_{1}}{\rho_{\mathrm{s}} L \cdot H \cdot b}$

where, $q_{f}$ is the total heat transferred by both convection and radiation from the fin surfaces (the two side-ends and the tip),

$$
q_{1}=\sum 2 \Delta x \Delta y\left[h_{i}^{p \cdot 1} \theta_{i, j}^{p \cdot 1}+\varepsilon \sigma\left(\left(\theta_{i, j}^{p_{i, 1}}+T_{, j}\right)^{4}-T_{0}^{4}\right)\right]
$$

and $q_{\max }$ is the maximum heat can be transferred by both convection and radiation from the fin surfaces (including the two ends and tip) of an ideal isothermal fin maintained at the base temperature, and it is simply calculated by replacing $\theta_{\mathrm{i}, \mathrm{j}}^{\mathrm{p}+1}$ by $\theta_{\mathrm{h}}$ in Eq. (18).

9) An effective limit of temperature ratio $\Gamma$ is assigned then the fin-height distribution is predicted and the corresponding $\xi$ is calculated

10) The effective temperature limit is altered till $\xi$ reaches its maximum. At this situation the optimum fin-height distribution $\mathrm{H}^{\prime}(\mathrm{x})$ is recorded.

The following flow chart represents diagrammatically the present procedure. 


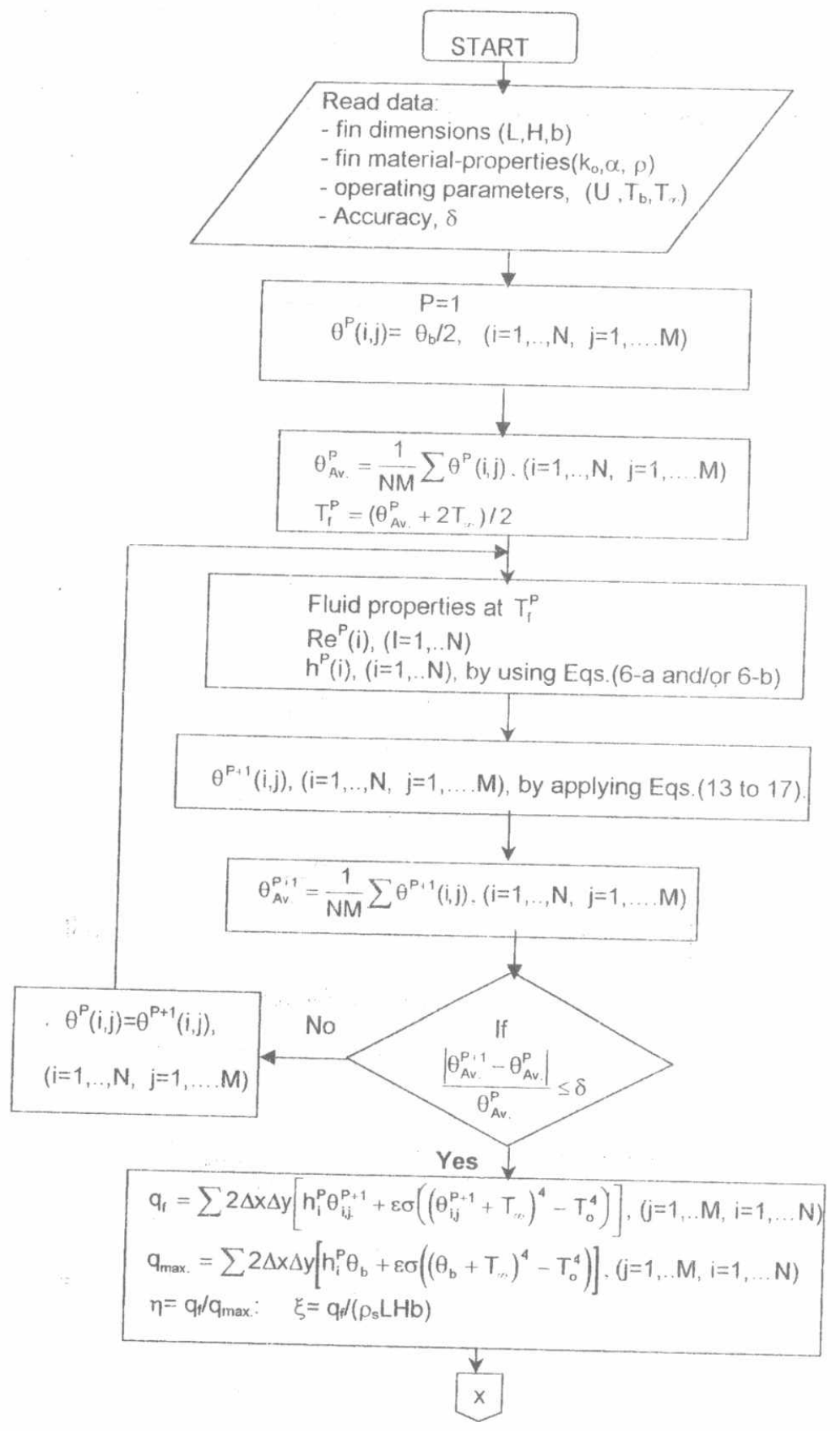




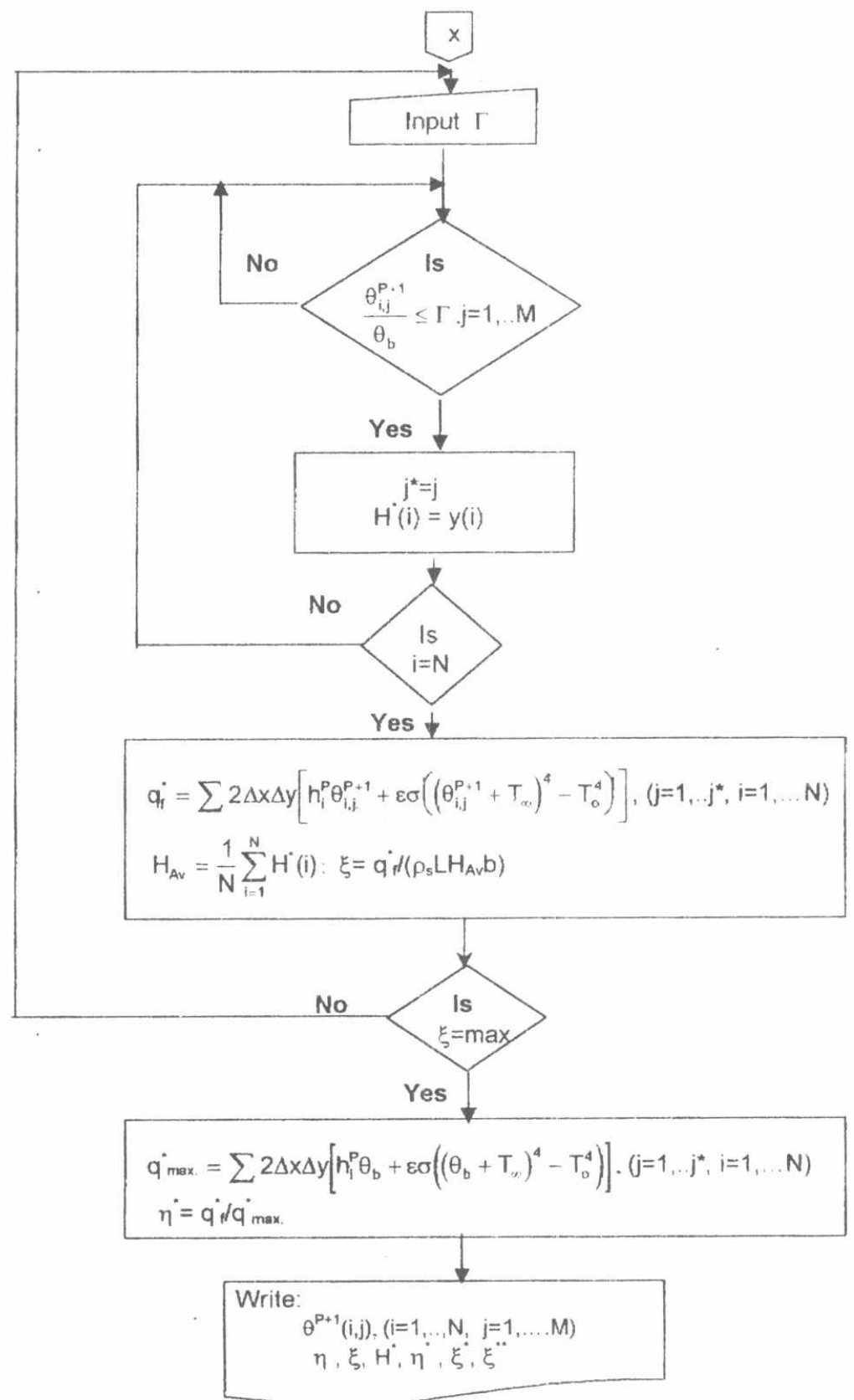




\section{Results and Discussion}

The validity of the present model and the accuracy of the predictions were evaluated via a comparison with some of the previously established results. The predicted fir efficiency for the limiting case of a one-dimensional fin with uniform heat transfer coefficient at constant base temperature was checked against that obtained for the the exact solution of solution of [16]. The comparisons show good agreement with as shown in assumption of pure the two different demperatures and at different flow velocities for varieties of fin materials. These predictions were plotted against the well-known term $L_{c}^{3}\left(\bar{h} / k_{0} A_{p}\right)^{\prime 2}$ as shown in Fig. (3), where $L_{c}=H+b / 2, A_{p}=L_{c} b$. It was found that the two-dimensional more realistic and more accurate) is lower than that obtained from the simple one-dimensional exact solution of [16]. This is due to the effect of the real simulation of the two-dimensional gradients of the fin surface temperature. Also, the overall efficiency of the convective-radiative fin was predicted taking into account the fin surface radiations and the results are shown in Fig.(4) The radiation effect was found to be more sensitive to the fin base temperature. This is naturally due to the principle dependent of the emissive heat flux with the fourth efficiency surface temperature. Although, there is an apparent reduction in the fin efficiency values, the overall fin heat dissipated is increased due to thermal radiation. An envelope of the predicted values between two limiting curves was observed as shown in Fig.(4). The upper limit, $\eta_{1}$, is valid for pure convective fins lower limit lower limit, $\eta_{11}$, is valid for convective-radiative fins working at elevated temperatures, These two limiting curves were then correlated as functions of the term $L_{c}^{3}\left(\bar{h} / k_{0} A_{p}\right)^{\prime 2}$ and the following two polynomials were obtained with a correlation factor of about 0.997 as: i)- for pure convective fins and for convective-radiative fins working at
$\theta_{b} \leq 100^{\circ} \mathrm{C}$;

$$
\eta_{1}=1.0-0.592 \psi+0.149 \psi^{2}-0.0169 \psi^{3}+7.0 \times 10^{-4} \psi^{4}
$$

ii)- for convective-radiative fins working at $\theta_{b},\left(300^{\circ} \mathrm{C}<\theta_{b} \leq 500^{\circ} \mathrm{C}\right)$;

where $\psi \quad$ is the term $L_{c}^{3}\left(\hat{h} / \mathrm{k}_{\mathrm{o}} A_{p}\right)^{1 / 2}$ and the correlations, Eqs. (19) and (20) are valid
only within a range of $\psi \leq 7.0$.

$$
\eta_{\text {III }}=0.857-0.542 \psi+0.157 \psi^{2}-0.021 \psi^{3}+10.3 \times 10^{4} \psi^{4}
$$

The temperature contours, isotherms, at different flow velocities were predicted and the effect asymmetric isotherms were obtained in general. This asymmetry is due to the contours was found when in the heat transfer coefficient. Further asymmetry in the contours was found when the flow velocity exceeds a critical range limited by 
$\operatorname{Re}_{L} \approx 5 \times 10^{5}$ beyond this range the flow regime will be changed from laminar to turbulent along the fin length.

The shape of the fin is considered an interesting parameter in any fin optimization criterion. Therefore, a method for reducing the volume of the straight fin and minimizing the operating costs was proposed by providing a fin-geometry with nonuniform height. This optimum fin-geometry is the trace of the effective fin heights at which a maximum amount of heat can be transferred per unit fin mass. These effective heights were predicted by calculating the $y$ values that having temperatures lower than a certain limit. This limit was estimated such that it maximizes the value of the heat dissipated per unit mass, $\xi$. It was found that, this limit appears within a range of a dimensionless temperature ratio $\left(0.05<\theta_{\text {eff }} / \theta_{b}<0.10\right)$ for the investigated ranges of the different parameters. Nonuniform fin-heights were predicted and Fig.(5) shows samples of the results for selected operating conditions. It was found that $H^{\prime}$ depends on $U, k$ and $T_{b}$ in general.

The present predictions of the effective heights were generalized to be valid for different operating conditions. This was carried out by correlating the predicted values using the least-squares method. The best fit of the present fin-height with a correlation factor of 0.965 was represented by a fifth order polynomial equation with a starting value $\lambda$, depending on both the fin material properties and the operating parameters as:

$$
\frac{H^{*}}{L}=\lambda+2.134\left(\frac{x}{L}\right)-10.674\left(\frac{x}{L}\right)^{2}+28.94\left(\frac{x}{L}\right)^{3}-33.575\left(\frac{x}{L}\right)^{4}+13.059\left(\frac{x}{L}\right)^{5}
$$

where $\lambda$ is a function of the reduced operating parameters represented by:

$$
\lambda=0.91\left(\frac{U}{U_{R}}\right)^{0.25}\left(\frac{k_{s}}{k_{R}}\right)^{0.5}\left(\frac{\theta_{b}}{\theta_{R}}\right)^{0.28}
$$

This correlation, Eq.(21), is valid with $\pm 10 \%$ maximum deviation with the present numerical results within the ranges of the following reduced parameters: $1<\frac{U}{U_{R}} \leq 50$, $1 \leq \frac{k_{s}}{k_{R}} \leq 4,1 \leq \frac{\theta_{b}}{\theta_{R}} \leq 20$ and $1 \leq \frac{\rho}{\rho_{R}} \leq 3.3$. Where, $U_{R}, \theta_{R}$ and $k_{R}$ are values for a reference fin which is a typical pure aluminum fin used as a heat sink for an electronic moduie, [13]. Values of $U_{R}=1 \mathrm{~m} / \mathrm{s}, \theta_{R}=25^{\circ} \mathrm{C}, k_{R}=237 \mathrm{~W} / \mathrm{m} . \mathrm{K}$ and $\rho_{R}=2702 \mathrm{~kg} / \mathrm{m}^{3}$, respectively, are assigned to these reference parameters

Based on these optimum fin-geometries the fin efficiencies were then recomputed and the results are shown in Fig.(6). It was found that the efficiency values exceed that of the one-dimensional fins. Therefore, according to these optimum fin-heights, it is possible to design more effective fins with less material and operate at a minimum pumping power consumption. Also, the present predictions for the efficiency of the improved fin-geometry were correlated in terms of the different parameters and the following correlation was obtained: 


$$
\eta^{\cdot}=0.41\left[\left(\frac{U}{U_{R}}\right)^{0.021}\left(\frac{k_{s}}{k_{R}}\right)^{0.262}\left(\frac{\theta_{b}}{\theta_{R}}\right)^{-0.197}\left(\frac{\rho}{\rho_{R}}\right)^{0.110}\right]
$$

This correlation is valid with $\pm 15 \%$ maximum deviation with the present predictions within the investigated ranges of the following reduced parameters: $1<\frac{U}{U_{R}} \leq 50$. $1 \leq \frac{k_{s}}{k_{R}} \leq 4,1 \leq \frac{\theta_{b}}{\theta_{R}} \leq 20$ and $1 \leq \frac{\rho}{\rho_{R}} \leq 3.3$.

The heat dissipated per unit mass for different fin materials, $\xi$, was computed per unit base temperature difference as: $\xi^{\circ}=\xi / \theta_{b}$ and the results are shown in Fig. (7-a). The values of $\xi$ were then generalized to cope with different fin materials. This was accomplished by introducing $\xi^{*}=\xi^{\circ}\left(\mathrm{k}_{\mathrm{s}} / \mathrm{k}_{\mathrm{R}}\right)^{0.5}$ as shown in Fig. $(7-\mathrm{b})$. The generalized heat dissipation $\xi^{*}$ was then correlated with the reduced flow velocity, $U / U_{R}$ and the following correlation was obtained:

$$
\xi^{*}=\left(\frac{\xi}{\theta_{b}}\right)\left(\frac{k_{s}}{k_{R}}\right)^{0.5}=1884.0+118.30\left(\frac{U}{U_{R}}\right)-3.90\left(\frac{U}{U_{R}}\right)^{2}+0.05\left(\frac{U}{U_{R}}\right)^{3} \text { W/kg.K }
$$

This correlation is valid with $\pm 2 \%$ maximum deviation within the investigated ranges of the reduced parameters.

The fin material volume reduction, in terms of volume saving ratio, was simply computed based on the concept of the optimum fin-geometry, VR $=(V-V) N$ where,$V$. is the improved fin volume and $V$ is the volume of a uniform height, conventional straight fin with an aspect ratio, $(H / L)=2$. Figure $(8)$ shows the volume saving ratio against the reduced flow velocity for the improved fin-geometrys for different fin materials at different base temperatures. It is evident that the fin volume reduction is dependent on the flow velocity and both the fin thermal conductivity and its base temperature. It was found that the value of VR decreases with either increasing the fin thermal conductivity or decreasing the operating temperature. As expected it was found that the saving in the fin material, represented by VR, increases with increasing the flow velocity. Fortunately, the use of such improved fin-geometry leads to an extremely saving in the flow pumping power for fin-systems operate at high flow velocities.

\section{CONCLUSIONS}

An analysis of a two-dimensional fin in a flow field with location dependent HTC was performed and the following concluding remarks were drawn:

1- The classical one-dimensional model is not valid in designing an effective fin working at elevated base temperature. 
2- Two-dimensional modeling of convective-radiative straight fins becomes more realistic and it gives precise predictions for geometries with Biot number based on the thickness $<0.1$.

3- The present correlations, Eqs. $(19,20)$ make it easy for the engineers to design traditional straight fins that actually fulfill the requirement of the fin heat duty.

4. The location dependency of the heat transfer coefficient affects the performance of the straight fins, in general. This effect becomes more significant when the working fluid velocity exceeds the laminar flow range, at $\mathrm{Re}_{\mathrm{L}}>5 \times 10^{5}$.

5- The proposed optimum fin-geometry for straight fin, represented by the correlation Eq.(21), makes it possible for the engineers to minimize both the material and the flow pumping power costs for thermal systems instrumented with fins.

6- The use of correlations, Eqs.(23-24) give the opportunity for engineers to design and use optimum fins with nonuniform fin-geometrys

\section{ACKNOWLEDGEMENTS}

This work has been done during the visit of the author to the Mechanical Eng. Dept., University of Strathclyde, UK. The author gratefully acknowledge all the members of the thermo-ftuid research group specially Dr. A. Gilchrist and Dr. K. Lee for their helpful supports.

\section{REFERENCES}

[1] Aziz, A., "Optimum Dimensions of Extended Surfaces Operating in A Convective Environment", Applied Mechanics Review, Vol. 45, No.5, pp. 155-173, May (1992).

[ 2] Murray, W.M., "Heat Transfer Through An Annular Disk or Fin of Uniform Thickness", Trans. ASME, J. of Appl. Mech., Vol. 60, Paper No. A78, (1938).

[3] Gardner, K.A., "Efficiency of Extended Surfaces", Trans. ASME, Vol. 67, pp.621631, (1945).

[ 4] Kraus, A., "Sixty-five Years of Extended Surfaces Technology", Appl. Mech. Rev., Vol.41, No.9, pp.321-364, (Sept., 1988).

[ 5] Leong, K.C. and Kooi, T.C., "Natural Convection from a Vertical Rectangular Fin", Experimental Heat Transfer, Vol.9, No.4, pp.287-303, (1996)

[ 6] Laor, K. and Kalman, H., "Performance and Optimum Dimensions of Different Cooling Fins with A Temperature-Dependent Heat Transfer Coefficient", Int. J. Heat Mass Transfer, Vol. 39, No.9, pp. 1993-2003, (1996).

[ 7] Yeh, R.H., "An analytical study of the optimum dimensions of rectangular fins and cylindrical pin fins", Int. J. Heat Mass Transfer, Vol.40, No.15, pp.36073615, (1997).

[ 8] Fabbri, G., "A genetic Algorithm for Fin Profile Optimization", Int. J. Heat Mass Transfer, Vol.40, No.9, pp.2165-2172, (1997).

[ 9] Garg, V.K. and Velusamy, K. "Heat Transfer Characteristics for A Flat Fin", ASME J. of Heat Transfer, Vol.108, pp.224-226, Feb.(1986).

[10] Shuja; S.Z. and Zubair, S.M., "Thermoeconomic optimization of constant crosssectional area fins", ASME, J. of Heat Transfer, Vol.119, pp.860-863, (1997). 
[11] Yu, L.T. and Chen, C.K., "Application of Taylor transformation to optimize rectangular fins with variable thermal parameters", Applied Mathematical Modeling, Vol.22, No.1-2, pp.11-21, (1998).

[12] Prasad, B.V. and Gupta, A. V., "Note on the performance of an optimal straight rectangular fin with a semicircular cut at the tip", Heat Transfer Engineering., Vol.19, No.1, pp.53-58, (1998).

[13] Morega, M. and Bejan, A., "Plate Fins with variable Thickness and Height for Air-cooled Electronic Modules", Int. J. Heat Mass Transfer, Vol. 37. Suppl.1. pp. 433-445, (1994).

[14] Kang, H.S., "Comparison of Performances of The Various Shapes of Asymmetric Fins", KSME International Journal, Vol.11, No.3, pp.311-318, (1997)

[15] Bejan, A., "Heat Transfer", Wiley, New York, (1993).

[16] Incropera, F.P. and DeWitt, D.P., "Fundamentals of Heat and Mass Transfer", $3^{\text {rd }}$ ed., John Wiley and Sons, New York, (1990). 


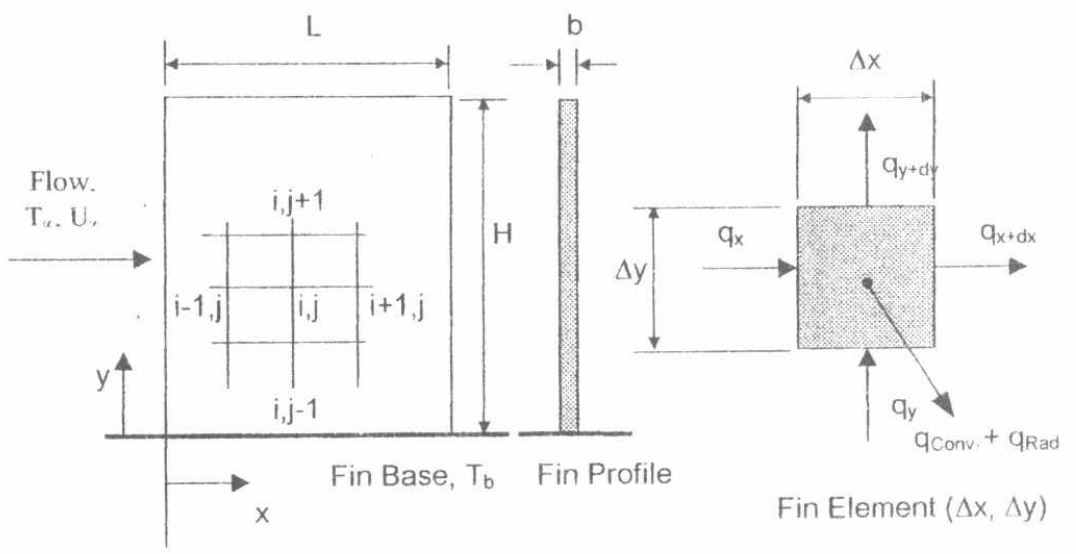

FIG. 1: Fin Geometry and Solution Domain

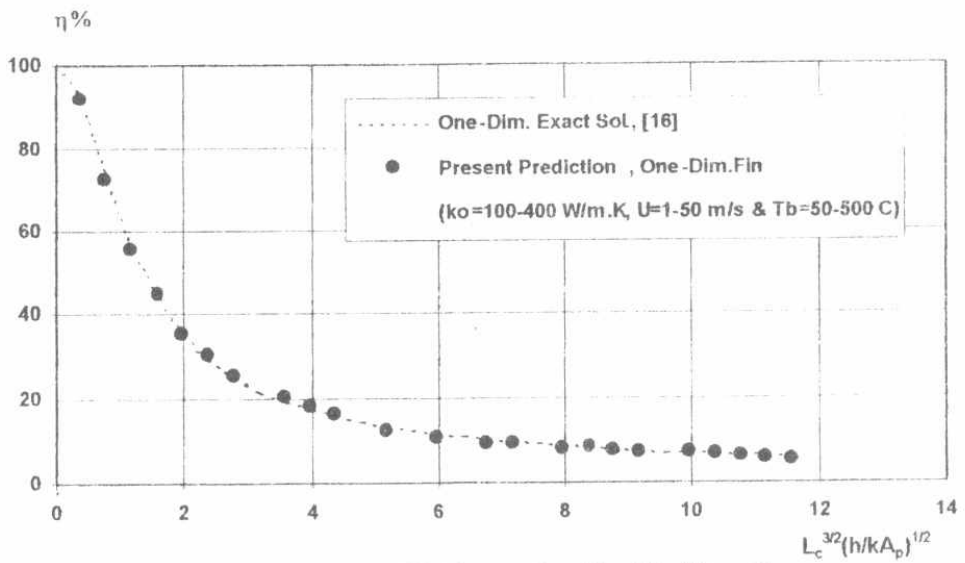

Fig.2. Predicted Fin-Efficiency for The Limiting Case of One-Dim. Fin with Uniform Convective HTC 


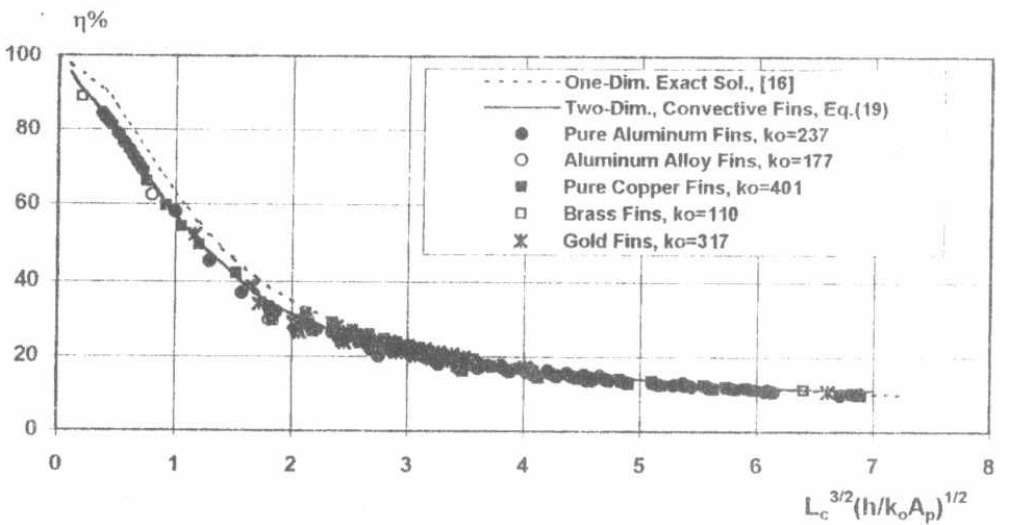

Fig. 3. Predicted fin efficiency for different fin-materials and dimensions, $\left(\mathrm{U}=1\right.$ up to $50 \mathrm{~m} / \mathrm{s}$ and $\mathrm{T}_{\mathrm{b}}=50$ up to $\left.500^{\circ} \mathrm{C}\right)$

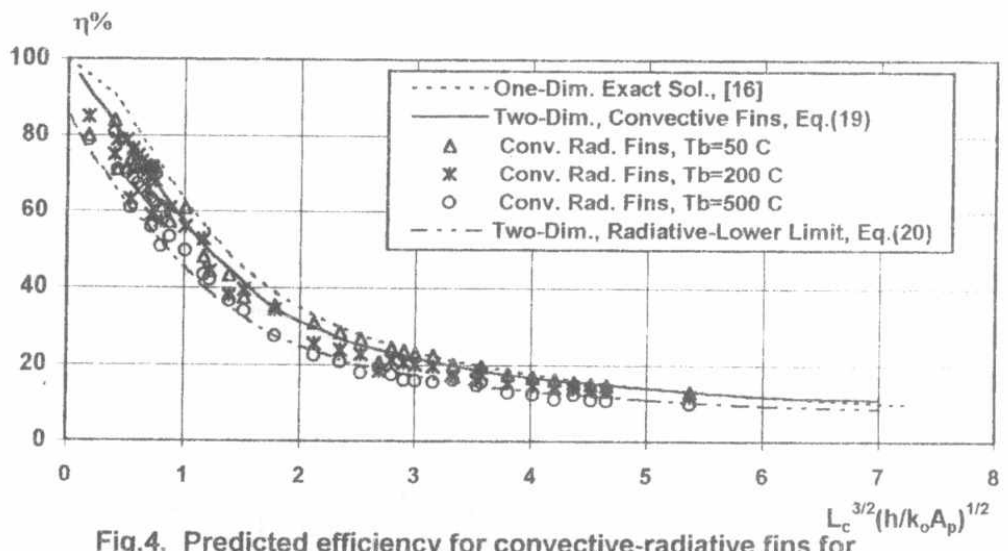

Fig.4. Predicted efficiency for convective-radiative fins for $\left(k_{o}=100-400\right.$ W/m.K, $U=1-50 \mathrm{~m} / \mathrm{s}$ and $T_{b}=50-500^{\circ} \mathrm{C}$ ) 


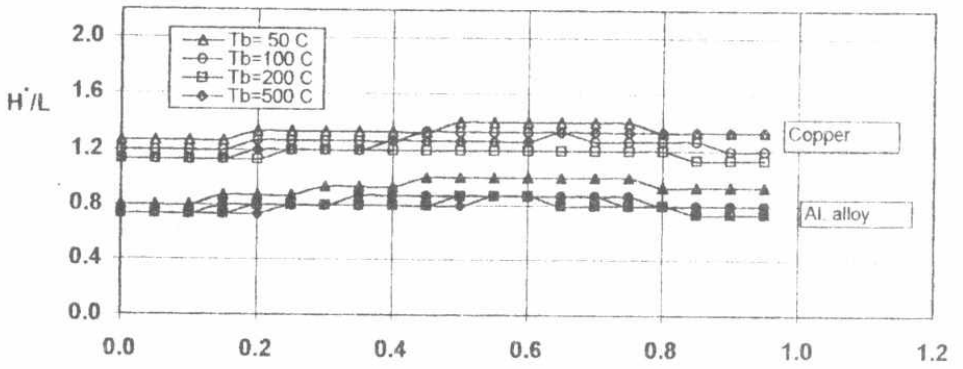

(a) Copper and aluminium alloy fins at $U=30 \mathrm{~m} / \mathrm{s}$

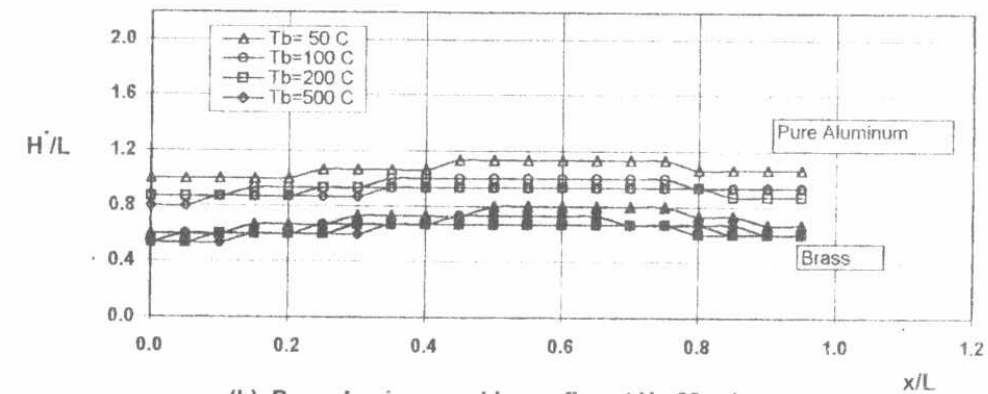

(b) Pure aluminum and brass fins at $U=30 \mathrm{~m} / \mathrm{s}$

Fig. 5. The predicted optimum fin-heights for different fin materials Bold symbols for materials with lower thermal conductivity

$\eta \%$

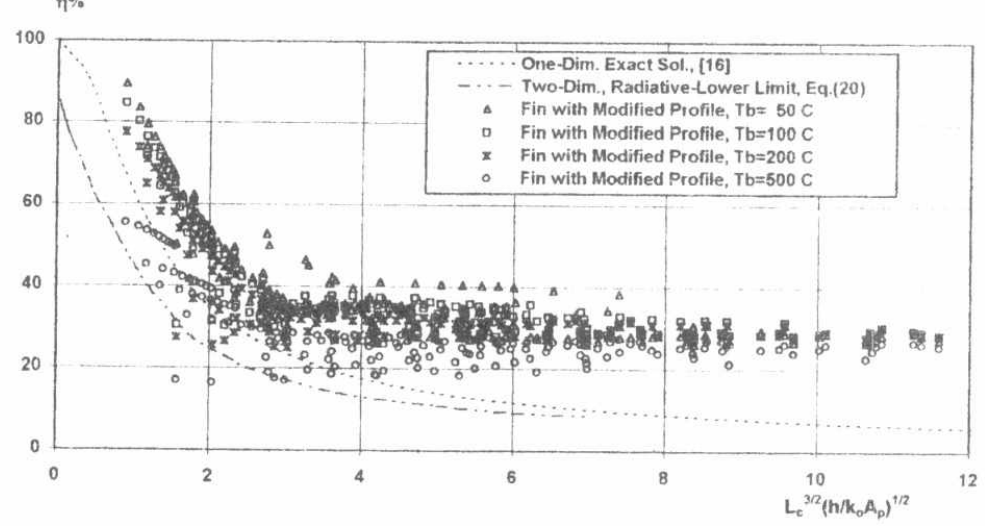

Fig. 6. Predicted efficiency for convective-radiative fins with optimum profiles $\left(k_{\mathrm{o}}=100-400 \mathrm{~W} / \mathrm{m} . \mathrm{K}, \mathrm{U}=1-50 \mathrm{~m} / \mathrm{s}\right.$ and $\theta_{\mathrm{b}}=25-500^{\circ} \mathrm{C}$ ) 

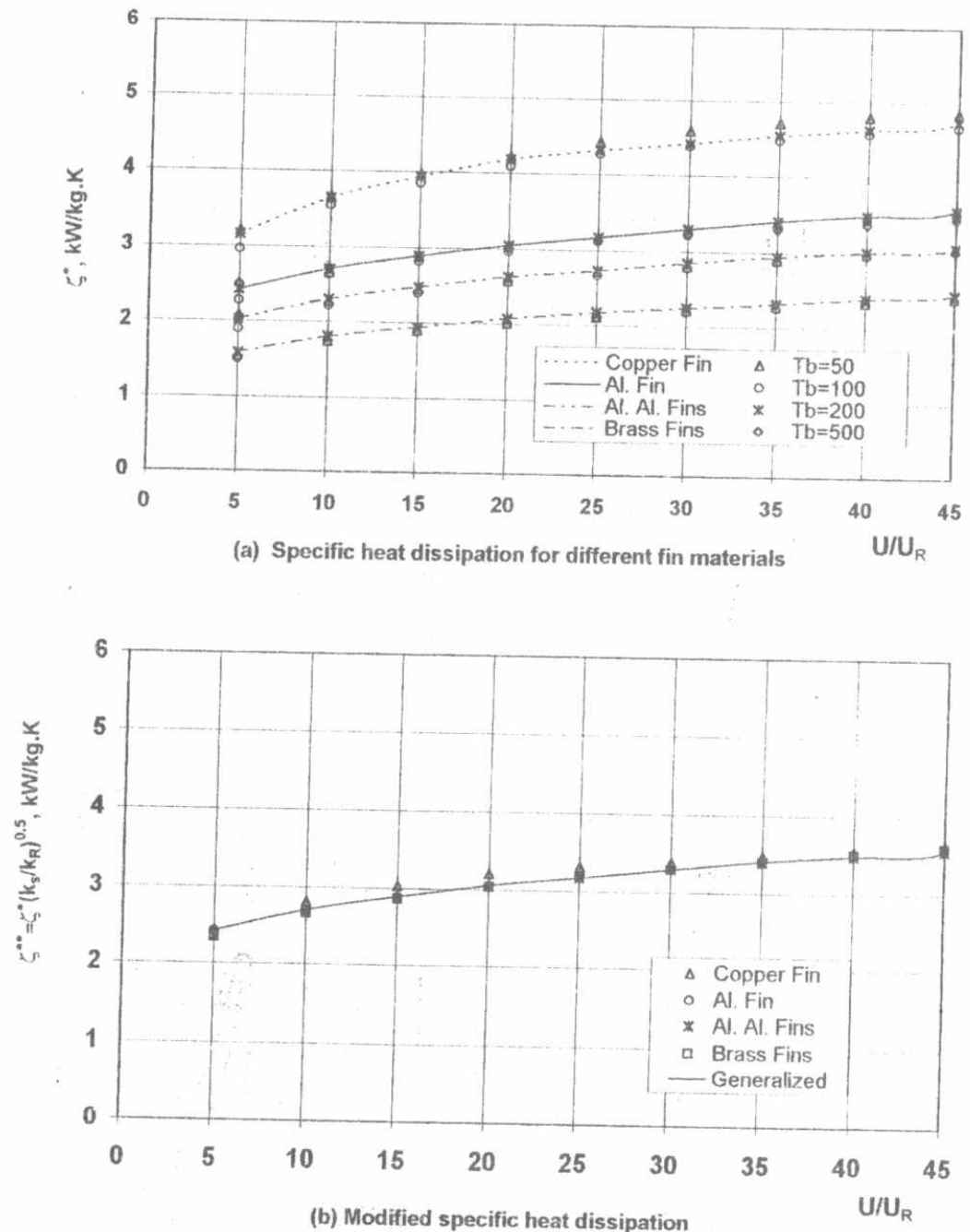

Fig .7. Heat dissipation per unit mass for the modified fin profile 

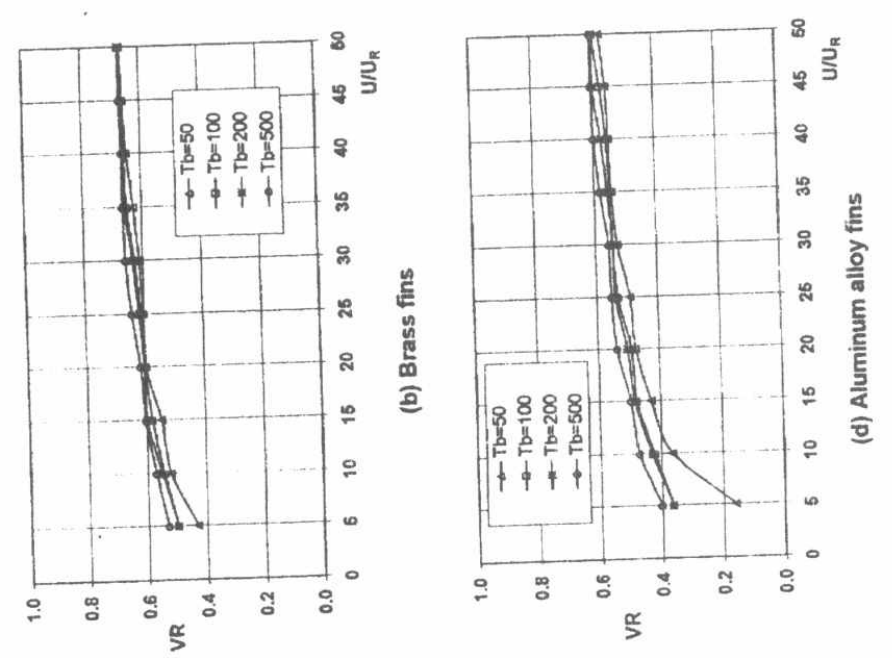

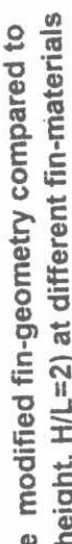
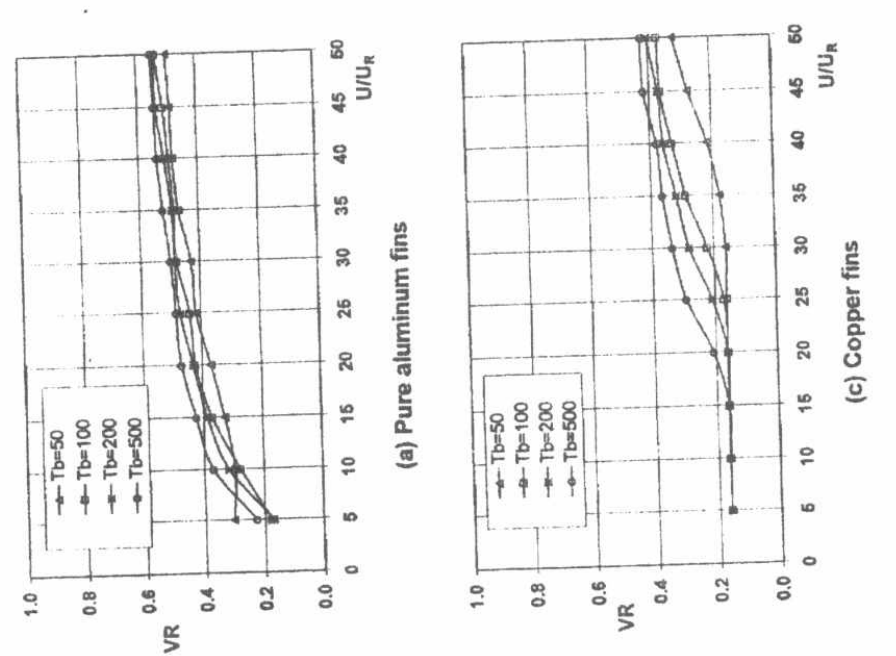

政 